\title{
Target Simulator Design based on ADSP-TS201
}

\author{
Feng Xiao ${ }^{1, a}$, Hang Chen ${ }^{2, b}$, Wei Wang ${ }^{3}$, Ye Zhang ${ }^{3}$ \\ ${ }^{1}$ Science and Technology on Underwater Acoustic Antagonizing Laboratory, Beijing, China \\ ${ }^{2}$ School of marine Science and Technology, Northwestern Polytechnical University, Xi'an, China \\ ${ }^{3}$ System Engineering Research Institute of CSSC, Beijing, China \\ ${ }^{a}$ xf_707@aliyun.com, ${ }^{b}$ chenhang@nwpu.edu.cn
}

Keywords: target signal simulator; ADSP-TS201; torpedo-homing system; parallel processing;

\begin{abstract}
A target simulator based on ADSP-TS201 was designed and implemented for researching and testing torpedo homing system. A multi-DSP parallel processing system was used as the hardware platform to generate element signals in real-time, and then the signals were provided for the torpedo homing system. In this paper, the architecture and functions of the target simulator were introduced firstly, and then the design and development of the hardware and software of the multiDSP parallel processing system were presented. At last, a performance testing for the implemented target simulator was carried out and the generated element signals were tested with the existing torpedo homing system. The results verified the effectiveness and correctness of the designed simulator and it is valuable for the torpedo homing research.
\end{abstract}

\section{Introduction}

Target simulator is an important supporting test equipment of sonar system, and its basic function is to generate various simulated signals which target echoes or emits for sonar probe hydrophone array to receive, but also to provide these signal to the sonar system for the purpose of sonar function testing or performance testing [1-3].

The early target simulator is application developed on the DOS operating system, which function is very poor. With the rapid development of computer technology, CPU speed has been greatly improved, the simulator which originally can only be achieved on an external circuit, can now be directly implemented using computer simulation technology [4].

With the appearance of high performance DSP, real-time signal processing system has found widespread applications. Especially in underwater acoustic detection system, real-time processing is the key, and the multi-DSP system is urgently needed with high speed A/D converter and vast data processing. The target simulator we designed is implemented on a multi-DSP platform. It uses some artificial signal generator to simulate sonar signals existed in real-world, such as echo, reverberation, target radiated noise, sea noise, and other sensor signals, and combine them to reach the same effect as the real world on the analog interface.

In this paper, a target simulator based on ADSP-TS201 was designed and implemented for researching and testing torpedo homing system. A multi-DSP parallel processing system was used as the hardware platform to generate element signals in real-time. The paper is organized as follows. Section 2 describes the architecture and functions of the target simulator in detail, including the design and development of the hardware and software of the multi-DSP parallel processing system. In Section 3, a performance testing for the implemented target simulator was carried out and the generated element signals were tested with the existing homing system. Finally, conclusions are drawn in Section 4.

\section{Target Signal Simulator}

According to the transmitting data rate and calculating time, TS201 is chosen to realize the system. The ADSP-TS201 TigerSharc processor is an ultrahigh performance, static superscalar processor optimized for large signal processing tasks and communications infrastructure. It supports 
floating-point and fixed-point processing, and also offers powerful features tailored to multiprocessing DSP systems through the external port and link ports. This multiprocessing capability provides the highest bandwidth for interprocessor communication, including up to 8 DSPs on a common bus, on-chip arbitration for glueless multiprocessing and link ports for point-topoint communication [5-7]. On the designed DSP board, each DSP has an independent external bus, and 256M bytes exclusive external memory (SDRAM). DSPs can exchange data through link ports, with the highest speed of $600 \mathrm{Mbytes} / \mathrm{s}$ and it does not take the time of DSP processors, so that data transfer between the DSPs will have higher transmission rate. To communicate with the host, all the DSPs and external memories connect to the CPCI (compact PCI) bus through shared local bus and PCI interface controller. In this way, the host can directly access any of the DSPs' internal and external memories.

The designs of target signal simulator include hardware part and software part. The parallel processing system with multi-DSP is used as the hardware platform in the presented target signal simulator, which is based on industrial personal computer with a DSP board and a D/A conversion board. In this paper, a set of application software is introduced mainly.

A set of application software for the target signal simulator with bottom-up layer style by means of module design comprises a main control software for the main control board, a DSP software for the DSP board, and a D/A conversion software for the D/A converter board. As up layer of management, the main control board software provides system management while DSP software and D/A conversion software perform system functions as bottom layer of task. The module design of the set of application software is described in Fig. 1.

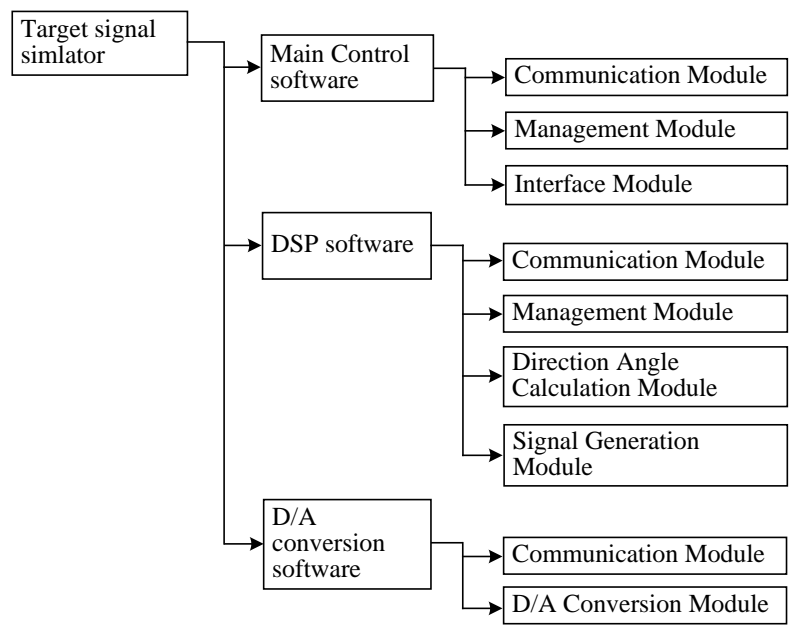

Figure 1 Architecture of set of target signal simulator softwares with modules

\section{Main Control Software}

Main control software offers the parallel processing system management and control to initial system, load DSP programs, interact with DSP board, and provide operating visualization.

\section{DSP Software}

DSP software is the key part to support parallel processing. According to the module design of the DSP software and the structure of DSP array, the generation of each type of target signals is map to each DSP, in order to achieve distributed parallel processing obtained with DSPs' cooperative working [8-10].

DSP software has three steps in data flow. The first step is to calculate the direction angle of target or jamming in certain location with underwater acoustic detection system, and then each type of target signals is generated respectively, where the target echo(or target radiant noise) and jamming signal is obtained with the direction angle, and others are isotropic. In the last step, these signals are added to form the received target signal. In the above, the task in the second step can also be performed simultaneously. Thus, the target generation with DSPs can be obtained with three pipelines. 


\section{Experiments and results}

Simulation experiments are carried out on the developed torpedo homing system.

In the experiments, the simulated target signals are generated with target signal simulator in active or passive mode. Figure 3 shows the waveforms of generated target signal from the target signal simulator in active mode and figure 4 shows the waveforms in passive mode. As huge data volume, only the first four channels of the target signal waveforms are displayed in Fig. 4 and also extracted on the scale of 100:1. From these two figures, we can only see the reverberation without the signals of target. Once the target occurs, figure 5 shows the combined target signals from target signal simulator in active mode. As shown in Fig. 5, the reverberation arrives first, and the target echo follows.

In Fig.5, the coordinates of the target and the torpedo in the torpedo homing system are as follows: target $(-3347.55,-5,-6386.53) \mathrm{m}$ and torpedo $(-2861.30,-5.08,-6503.15) \mathrm{m}$. The vehicle velocity of the target and the torpedo is $18 \mathrm{kn}$ and $50 \mathrm{kn}$. The current course of the target and the torpedo is 270 deg and $284.01 \mathrm{deg}$. The real value of the horizontal angle, vertical angle, and relative distance of the target calculated in terms of the coordinates of the target and the torpedo are -13.49 deg, $0.01 \mathrm{deg}$, and $500.04 \mathrm{~m}$, respectively.

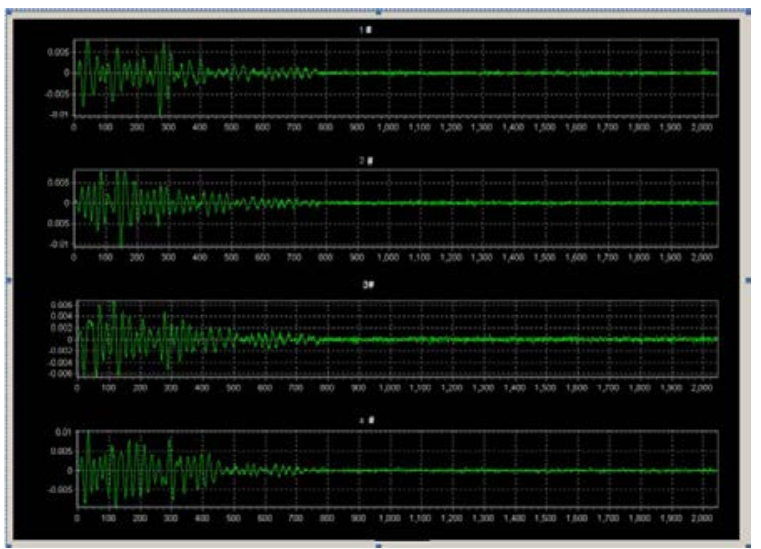

Fig. 3 Waveforms of generated target signals from target simulator in active mode

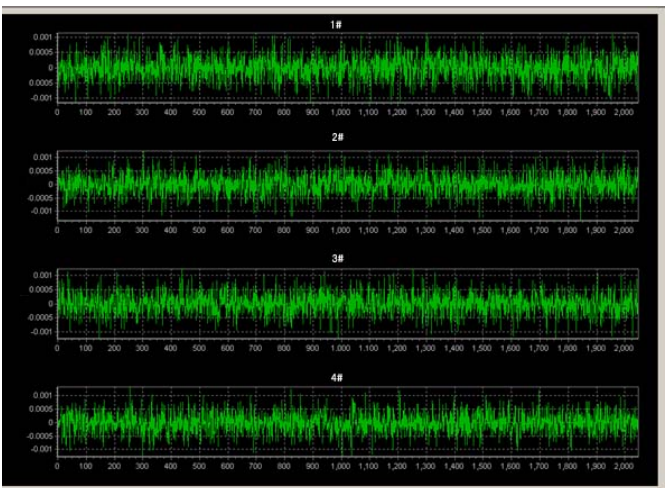

Fig. 4 Waveforms of generated target signals from target simulator in passive mode

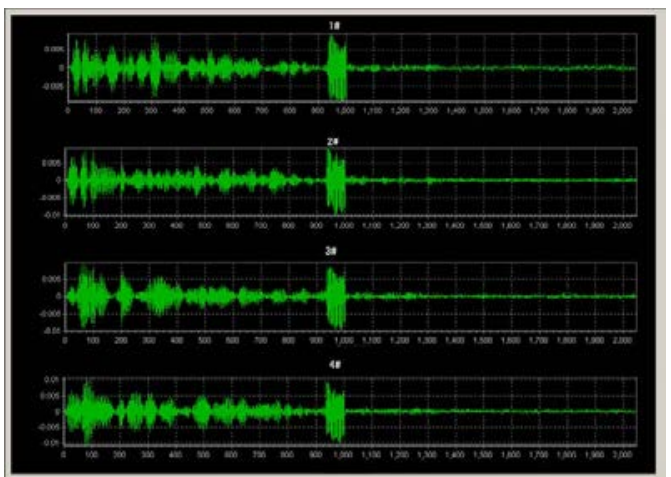

Fig. 5 Waveforms of combined target signals with reverberation from target simulator in active mode 
In order to simulate the process of acoustic countermeasure in our torpedo homing system, the target simulator can also produce some extra signals of target such as the acoustic decoy. Figure 6 shows the waveforms generated from the target simulator, which including target signals, reverberation, and the acoustic decoy. As shown in Fig. 6, the reverberation arrives first, and the signals of acoustic decoy follows, behind it there is the signals of target echo.

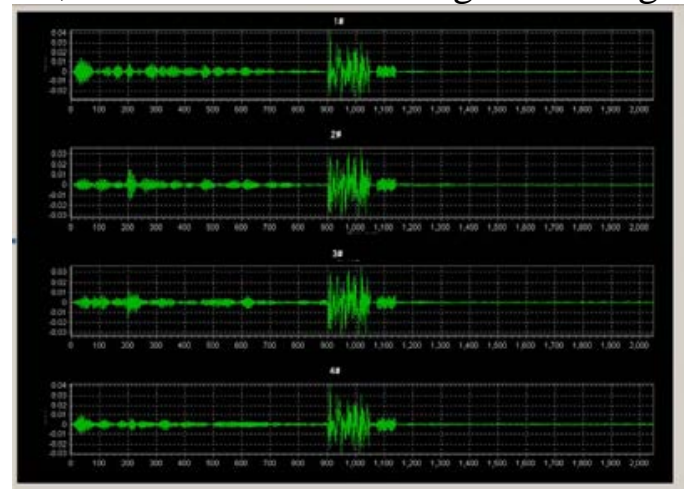

Fig. 6 Waveforms of combined target signals with reverberation and acoustic decoy from target simulator in active mode

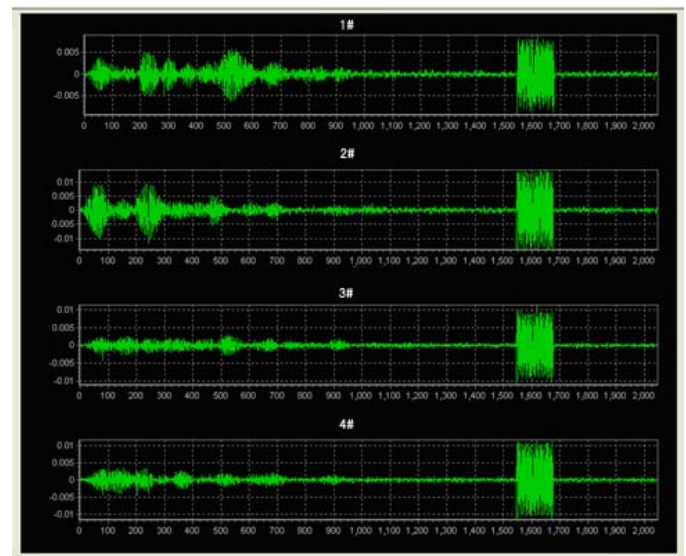

Fig. 7 Waveforms of beam signals with signal processing system

Figure 7 shows the beam signals from signal processing system in our torpedo homing system. Split beamforming is used in the signal processing system. As shown in Fig. 7, the first two channels are signals of left beam and right beam in horizontal direction and the last two channels are signals of up beam and bottom beam in vertical direction. Meanwhile, the improvement obtained with beamforming is achieved obviously.

As shown in Fig. 7, the estimated values of the horizontal angle, vertical angle, relative distance, and relative speed can be obtained from the torpedo homing system.

\section{Conclusions and future work}

In this paper, a target simulator based on ADSP-TS201 was designed and implemented for researching and testing torpedo homing system. A multi-DSP parallel processing system was used as the hardware platform to generate element signals in real-time, and then the signals were provided for the torpedo homing system. A performance testing for the implemented target simulator was carried out and the generated element signals were tested with the existing torpedo homing system. The results verified the effectiveness and correctness of the designed simulator and it is valuable for the torpedo homing research.

\section{References}

[1] Zheng Z., Li J., WU S., "Design of a Radar Signal Simulator Based on Virtex- II Series FPGA”, Journal of Beijing Institute of Technology, vol. 15, no. 1, 2006, pp.106-110.. 
[2] GUO J.Y., MA M. and REN Q., "Design and implementation of shore-based radar simulator." Modern Electronics Technique, vol. 14, 2012.

[3] LI, L., Wu Y., and CAI H.., "Real-time software development for sonar array signal simulator." Technical Acoustics, vol. 3 , 2004.

[4] G. Yang and Q. Yuan, "Target Simulator Design Based on Virtual Instrument Technology.” 2010 International Conference on Electrical and Control Engineering, 2010, pp. 2282-2231.

[5] Lion Technology Inc, http://www.lionteck.net, 2010.

[6] ADSP-TS201 Tiger SHARC Processor Hardware Reference (Revision 1.1). USA: Analog Devices Inc, 2004.12.

[7] ADSP-TS201 Tiger SHARC Processor Data Sheet (Revision C). USA: Analog Devices Inc,2006.12

[8] MING X, ZHANG G, Lü Q. "Multi-DSP Parallel Processing Technique of Hyperspectral RX Anomaly Detection,” Spectroscopy and Spectral Analysis, vol.34, no.5, pp. 1383-1387.

[9] Thirer, N., Souhami, A., "Parallel processing for a DSP application using FPGA", IEEE Convention of Electircal and Electronics Engineers in Israel, 2006, pp. 389-392..

[10] Li B., Ma X., Yan S. and Yang L., "Design of Multi-DSP sonar array processing system based on VPX standard and implementation of broadband beamforming”, International Journal of Digital Content Technology and its Applications, vol.5, no. 12, 2011, pp.362-371. 